Research Paper

\title{
The Extracts of Astragalus membranaceus Inhibit Melanogenesis through the ERK Signaling Pathway
}

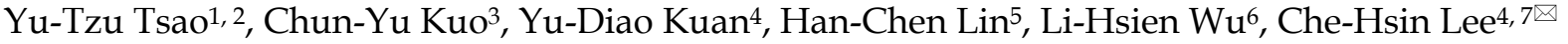 \\ 1. Division of Nephrology, Department of Medicine, Taoyuan General Hospital, Taoyuan 330, Taiwan; \\ 2. Institute of Clinical Medicine, National Yang-Ming University, Taipei 11221, Taiwan; \\ 3. Graduate Institute of Basic Medical Science, School of Medicine, China Medical University, Taichung 404, Taiwan; \\ 4. Department of Biological Sciences, National Sun Yat-sen University, Kaohsiung 804, Taiwan; \\ 5. Department of Anatomy, School of Medicine, College of Medicine, Kaohsiung Medical University, Kaohsiung, Taiwan; \\ 6. Department of Public Health, China Medical University, Taichung 404, Taiwan; \\ 7. Department of Medical Research, China Medical University Hospital, China Medical University, Taichung 40402, Taiwan.
}

$\square$ Corresponding author: Dr. Che-Hsin Lee, Department of Biological Sciences, National Sun Yat-sen University, Kaohsiung, Taiwan, 70 Lienhai Rd. Kaohsiung 80424, Taiwan. E-mail: chlee@mail.nsysu.edu.tw

(c) Ivyspring International Publisher. This is an open access article distributed under the terms of the Creative Commons Attribution (CC BY-NC) license (https://creativecommons.org/licenses/by-nc/4.0/). See http://ivyspring.com/terms for full terms and conditions.

Received: 2017.03.30; Accepted: 2017.07.05; Published: 2017.09.03

\begin{abstract}
Melanin is a normal production protecting skin from environment-causing damage. Plants produce some agents in response to their environment. These agents could be applied in cosmetic production. Some Chinese herbals have immunomodulatory activities and modulate the symptoms of several diseases. Melanogenesis represents a complex group of conditions that are thought to be mediated through a complex network of regulatory processes. Previously, some studies found that the extracts of Astragalus membranaceus (PG2) regulated immunity and supported hematopoiesis. Herein, we want to determine the molecular mechanisms by which PG2 inhibits melanogenesis in B16F10 melanoma cells. The cellular melanin contents and expression of melanogenesis-related protein, including microphthalmia associated transcription factor (MITF) and tyrosinase were significantly reduced after PG2 treatment. Moreover, PG2 increased phosphorylation of ERK, without affecting phosphorylation of $\mathrm{p} 38$. These results suggested that PG2 as a new target in reducing hyperpigmentation through the ERK signal pathway. PG2 has potential for cosmetic usage in the future.
\end{abstract}

Key words: Melanin; Astragalus membranaceus; PG2; melanogenesis.

\section{Introduction}

The degree and distribution of melanin pigmentation is the main regulator of the hair and skin color. Moreover, melanin plays important photo-protective roles in the carcinogenic and the deleterious effects of ultraviolet radiation of solar light. In European, irregular hyperpigmentation may result in various dermatological disorders, including melasma and age spots. In Asia, people make skin whiter is extended. Past studies focused on the screening of natural compounds from plant sources for anti-melanogenic agent $[1,2]$. The a-melanocytes stimulating hormone (a-MSH) binds the melanocortin-1 receptor (MC1R), induces cAMP pathway and results in melanin synthesis. The microphthalmia-associated transcription factor (MITF) regulates enzymes required for melanin synthesis including tyrosinase, tyrosinase-related protein 1(TRP1), and tyrosinase-related protein 2 (TRP2). Tyrosinase is an important enzyme, as it regulates the rate limiting steps in melanogenesis. Plants can produce some compounds for cosmetic applications. Some agents have immunomodulatory activities and modulate the inhibition of melanogenesis [3]. Traditional Chinese medicine, an important aspect of alternative medicine. Astragalus membranaceus is a medicinal herb that is widely used in the treatment of inflammatory diseases, tumors, radical scavenger activity, various cardiovascular 
diseases, and neuroprotective activity [4-6]. A polysaccharide isolated from the radix of $A$. membranaceus, called PG2, used in traditional Chinese medicine, with potential hematopoiesis inducing and immunomodulating activities. PG2 extracted from $A$. membranaceus has been demonstrated as a novel alternative medicine for cancer patients $[7,8]$. In this study, we sought to investigate an anti-melanogenic signaling pathway in a-MSH-treated B16F10 melanoma cells by treating PG2. To improve the efficacy and safety of PG2, a further understanding of mechanism of PG2 anti-melanogenesis is required. Our studies may offer new roads to increase the anti-melanogenic potential of PG2.

\section{Materials and Methods}

\section{PG2 and cells}

PG2 is a botanical drug and purified from $A$. membranaceus. PG2 are available from PhytoHealth Corp (Taipei, Taiwan). Dulbecco's modified Eagle's medium containing $10 \%$ fetal bovine serum, $1 \%$ glutamine, and $50 \mu \mathrm{g} / \mathrm{ml}$ gentamicin was used for culturing murine B16F10 melanoma cells at $37^{\circ} \mathrm{C}$ in $5 \% \mathrm{CO} 2$ condition.

\section{Cell proliferation assay}

Cells $\left(10^{5} /\right.$ well $)$ were treated with various concentration of PG2 in serum- free medium for $24 \mathrm{~h}$. Cell proliferation was assessed by Cell Counting Kit-8 (Sigma-Aldrich, St. Louis, MO, USA) according to the manufacturer's instructions [3].

\section{Melanin content assay}

The cells were harvested and lysed in cold lysis buffer (20 mM sodium phosphate $\mathrm{pH}$ 6.8, 1\% Triton $\mathrm{X}-100,1 \mathrm{mM}$ PMSF, and $1 \mathrm{mM}$ EDTA) at the end of experiment. The melanin pellets were dissolved in Soluene-350 (Perkin-Elmer, Waltham, MA, USA) for $15 \mathrm{~min}$ at $100^{\circ} \mathrm{C}$ and centrifuged at $15,000 \mathrm{~g}$ for 15 $\mathrm{min}$. The absorbance at $500 \mathrm{~nm}$ was measured [9]. The bicinchoninic acid (BCA) protein assay was used for protein content (Pierce Biotechnology, Rockford, IL, USA). The PD98059 (50 $\mu \mathrm{M})$ (Sigma-Aldrich) were pretreated to cells for $1 \mathrm{~h}$, then PG2 was added to cells. PG2-treated or untreated cells were lysed in cold lysis buffer and protein lysis was harvested.

\section{Western blot analysis}

Proteins from cellular lysis were fractionated on SDS-PAGE, transferred onto Hybond enhanced chemiluminescence nitrocellulose membranes (Amersham, Little Chalfont, UK), and probed with primary antibodies against tyrosinase (Santa Cruz Biotechnology, Santa Cruz, CA, USA), MITF (Merck Millipore Darmstadt, Germany), ERK (Santa Cruz
Biotechnology), phosphor-ERK (Santa Cruz Biotechnology), p38 (Santa Cruz Biotechnology), phosphorylated-p38 (Santa Cruz Biotechnology) or monoclonal antibodies against $\beta$-actin (AC-15, Sigma Aldrich). Horseradish peroxidase-conjugated secondary antibodies were used, and protein-antibody complexes were visualized by enhanced chemiluminescence system. The signals were quantified with Image software (rsbweb.nih.gov/ij/) [10].

\section{Statistical analysis}

All data were expressed as mean \pm standard deviation (SD). The one- way ANOVA test was used to determine differences between groups. Any $P$ value less than 0.05 is considered statistically significant.

\section{Results}

\section{Evaluation of anti-melanogenic activity of PG2 in vitro}

The anti-melanogenic activity of PG2 was evaluated by using mouse B16F10 melanoma cell system. As shown in Fig. 1, the cell survival and melanin content in B16F10 cells were measured after PG2 treatment. The significant cytotoxicity were not observed in PG2- treated cells (1-100 ng/ml) (Fig. 1A). The melanin content significant upregulated in a-MSH-treated cells. The melanin content of a-MSH-stimulated B16F10 cells was reduced after PG2 treatment (Fig. 1B). Treatment with PG2 resulted in a significant and dose-dependent decrease in the melanin content of B16F10 cells (Fig. 1C). These results demonstrated that PG2 inhibited the melanogenesis.

\section{PG2 dose-dependently down-regulated the expression levels of tyrosinase and MITF}

We fist examined the expression levels of tyrosinase and MITF by Western blotting to elucidate the mechanisms underlying the anti-tyrosinase and anti-melanogenic activity of PG2. MITF transcriptionally regulated the protein level of tyrosinase. The tyrosinase and MITF are regarded as target enzymes for anti-melanogenesis. PG2 dose-dependently reduced the expression of tyrosinase and MITF in Fig. 2.

\section{Effects of PG2 on the mitogen-activated protein kinase (MAPKs) signaling pathway}

MAP kinase are involved in melanogenesis. The MAP kinase family including ERK and p38 has been reported in hyperpigmentation [11, 12]. The ERK signaling can down-regulate the melanin synthesis. Furthermore, up-regulation of phosphorylation of p38 is related to the production of melanin. We examined 
the involvement of MAPK signaling pathway in the melanogenic effect of PG2 by immunoblotting. In this study, the a-MSH significant inhibited the ERK signaling pathway (Fig. 3). However, PG2 increased the phosphorylation of ERK in the a-MSH-treated cells without influencing p38 pathway. However, ERK phosphorylation significantly decreased after a-MSH treatment (Fig. 3). In order to evaluate the relationship between the anti-melanogenesis effect of PG2 and the ERK pathway, PD98059, a selective inhibitor of ERK, was used the stimulatory effects of a-MSH on MITF and tyrosinase as shown in Fig. 4A.
PD98059 significantly reduced the expression of ERK phosphorylation. PG2 reduced the expression of tyrosinase and MITF in a-MSH-PD98059-PG2 group compared with those in a-MSH-PD98059 group. Meanwhile, the melanin increased after PD98059 treatment (Fig. 4B). PG2 slightly reduced melanogenesis after a-MSH and PD98059 treatment. The ERK signaling inhibitors blocked the hypopigmenting effects induced by PG2. These results suggest that PG2 increases ERK signaling to inhibit melanogenesis.

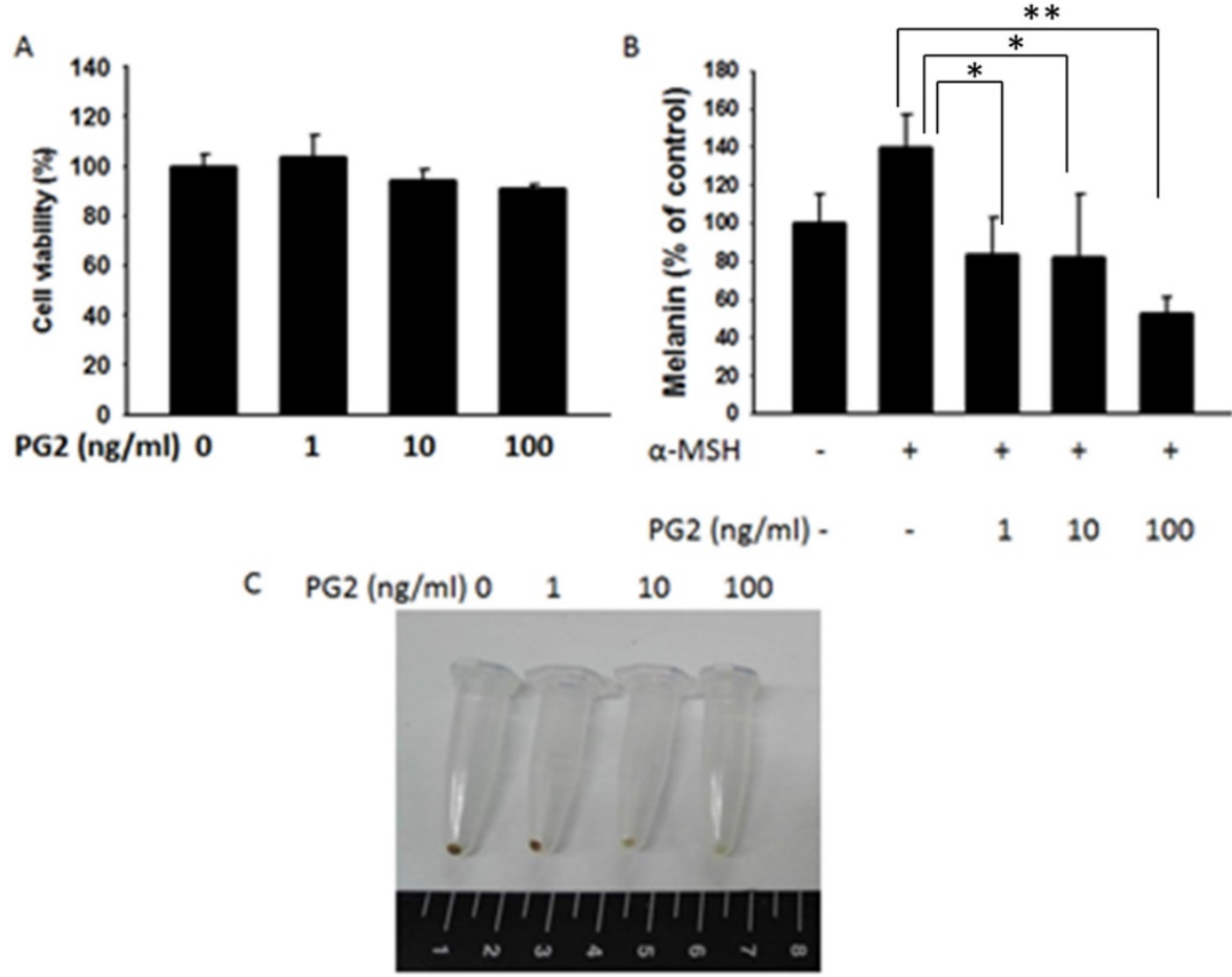

Figure 1. Effects of PG2 on cell viability and melanin production in B16F10 cell. Cells were treated with indicated concentrations of PG2 for $24 \mathrm{~h}$. (A) Cell viability was measured by cell proliferation assay. (B) Effect of PG2 on cellular melanin content. (mean \pm SD, $n=6$ ) (C) Photograph of precipitated B16F10 melanoma cells. Cells were incubated for $48 \mathrm{~h}$ with $(1-100 \mathrm{ng} / \mathrm{ml})$ and without PG2. *, $P<0.05 ; * *, P<0.01$.

\begin{tabular}{|c|c|c|c|c|c|}
\hline$\alpha-\mathrm{MSH}$ & - & + & + & + & + \\
\hline PG2 (ng/ml) & 0 & 0 & 1 & 10 & 100 \\
\hline \multirow[t]{2}{*}{ Tyrosinase } & exinisis & ates & semiles & $-60.00=$ & $T$ \\
\hline & 1.00 & 1.11 & 0.88 & 0.76 & 0.54 \\
\hline \multicolumn{6}{|l|}{ MITF } \\
\hline & 1.00 & 1.05 & 0.71 & 1.37 & 1.20 \\
\hline
\end{tabular}

Figure 2. The expression levels of tyrosinase and MITF after PG2 treatment. Cells were treated with PG2 at the concentration of 1,10 or $100 \mathrm{ng} / \mathrm{ml}$ for $24 \mathrm{~h}$. The protein expression was determined by Western blotting. Inserted values indicated relative proteins expression in comparison with $\beta$-actin. 


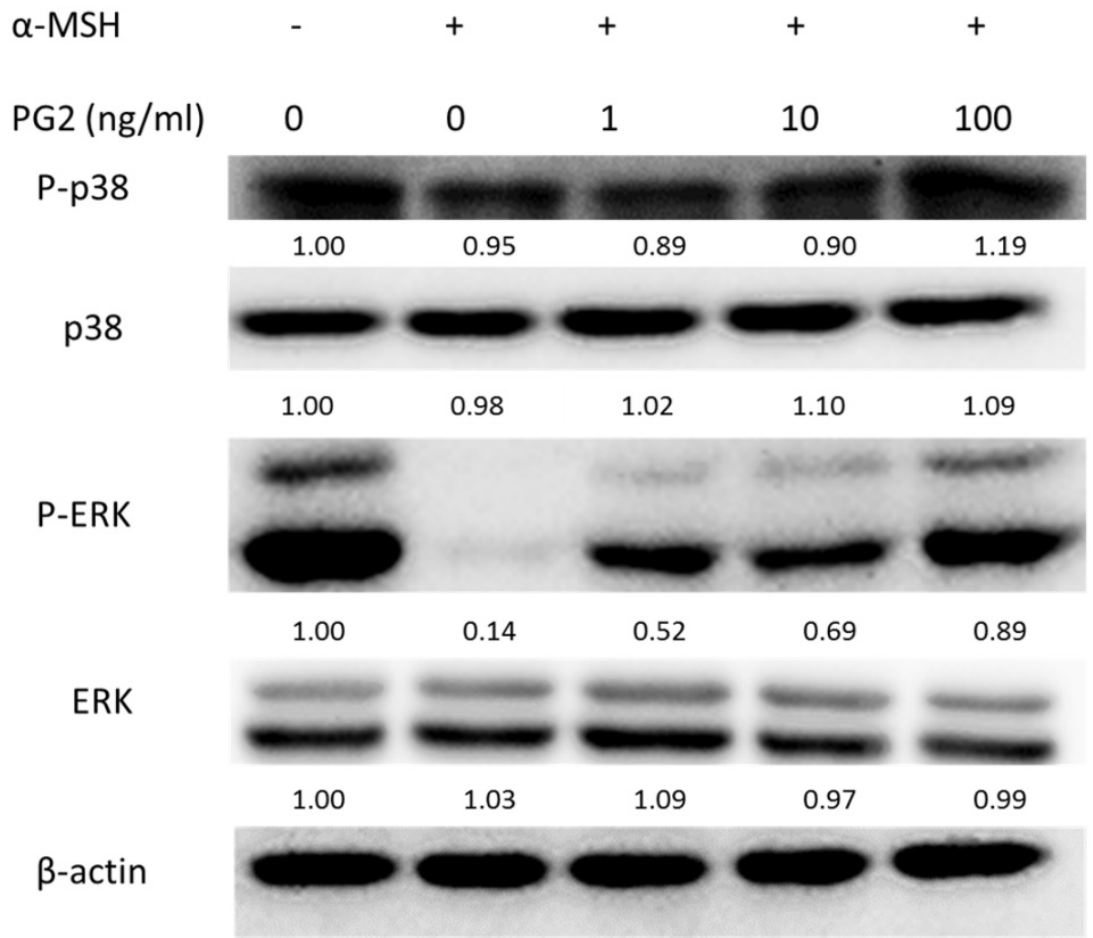

Figure 3. Effect of PG2 on the expression levels of MAPKs and phosphorylated-MAPKs in cells. Cells were treated with PG 2 at the concentration of 1,10 or 100 $\mathrm{ng} / \mathrm{ml}$ for $24 \mathrm{~h}$. The protein expression was determined by immunoblotting. Inserted values indicated relative proteins expression in comparison with $\beta$-actin.

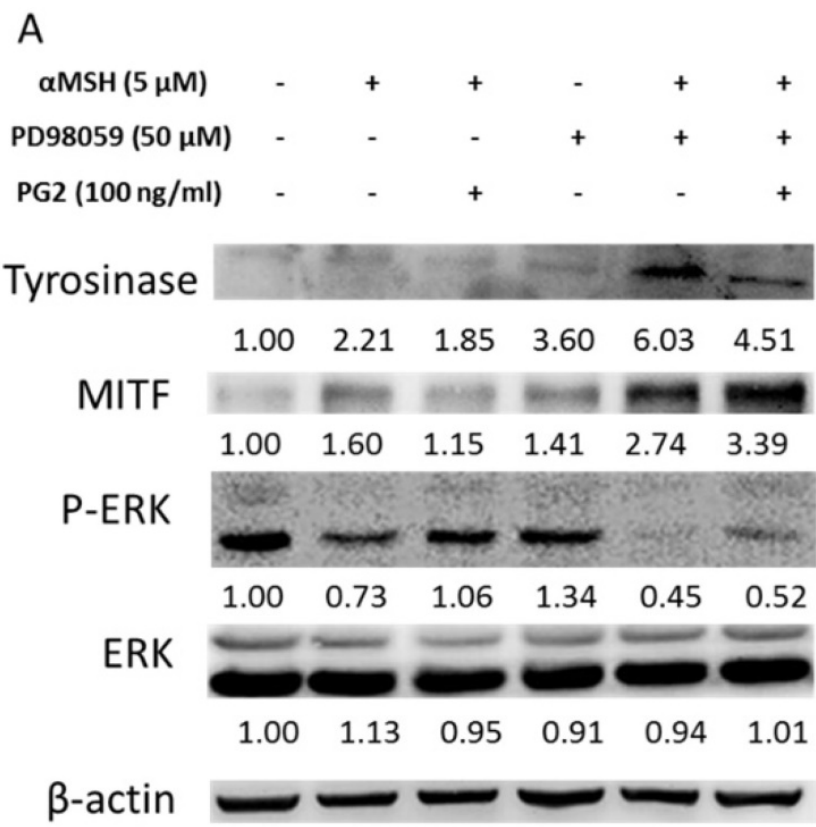

B

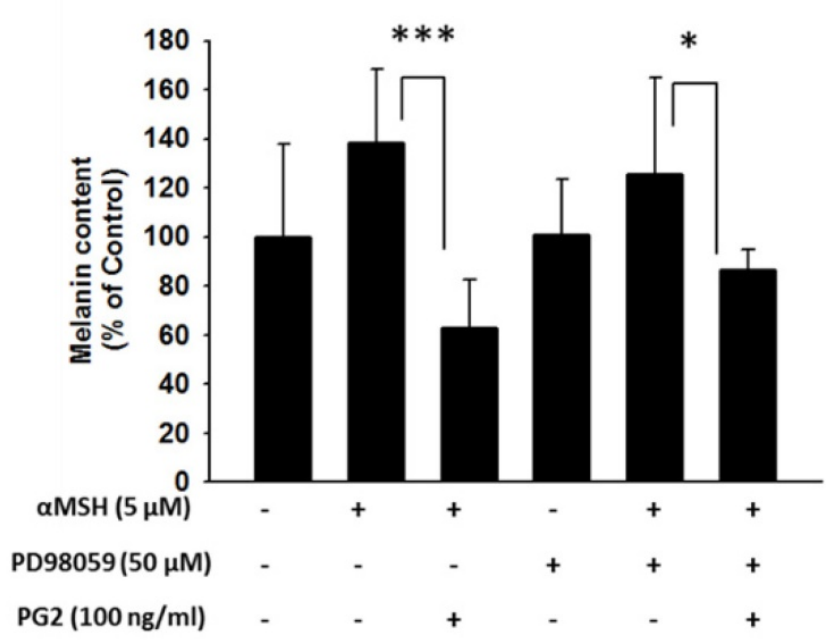

Figure 4. PG2 inhibited the production of melanin through ERK pathway. B16F10 cells were treated with PG 2 at the concentration of $100 \mathrm{ng} / \mathrm{ml}$ for $24 \mathrm{~h}$ and PD98059 $(50 \mu \mathrm{M})$ for $1 \mathrm{~h}$. (A) The protein and (B) melanin expression were measured (mean $\pm S D, n=6)$. The expression of $\beta$-actin served as the quantitative control. Inserted values indicated relative proteins expression in comparison with $\beta$-actin. *, $P<0.05 ; * * *, P<0.001$.

\section{Discussion}

PG2 is a bioactive extract of A. membranaceus which is believed to improve palliative treatment in advanced cancer patients [7]. In advanced cancer patients, PG2 is safe and effective botanical-derived drug. In this study, PG2 significantly inhibited the melanin production in B16F10 cells. Tyrosinase and MITF were reduced after treatment with PG2, as assessed by Western blot analysis in a dose-dependent manner. Herein, we demonstrated that PG2 could inhibit melanin production by 
up-regulating to ERK signaling pathway after a-MSH treatment. PG2 could be an effective and safe agent for using in cosmetics. The inhibition of p38 suppressed activation of cAMP response element-binding protein (CREB) in melanogenesis [12]. In this study, PG2 did not significantly influence the phosphorylation of p38. The activation of ERK signal pathway induced the degradation of MITF [13]. However, the involvement of ERK signaling pathway was confirmed by the treatment of PD98059 in PG2-treated B16F10 cells. Pleiotrophin increased the level of ERK phosphorylation and inhibited the production of melanin [14]. PG2 could dramatically activate ERK signaling pathway to regulate melanin synthesis. The phosphorylation of ERK is significantly observed when B16F10 cells are treated with PG2. Consistent with the previous finding, down-regulation of ERK signaling pathway by a chemical inhibitor reduced the anti-melanogenic effect of PG2. The molecular weights of PG2 is between 20 to 60 kilodalton. PG2 contains a-1,4-linked glucan, arabinogalactans, rhamnogalacturonans and arabinogalactan protein. Recent studies have suggested that PG2 could modulate immunological function and down-regulate P-glycoprotein expression in tumor [8]. By taking advantages of the pleiotropic activities of PG2, we can come to a conclusion that PG2 appears to hold promise for the treatment of melanogenesis.

\section{Abbreviations}

MITF: microphthalmia associated transcription factor a-MSH: a-melanocytes stimulating hormone

MC1R: melanocortin-1 receptor

TRP1: tyrosinase-related protein 1

TRP2: tyrosinase-related protein 2

MAPK: mitogen-activated protein kinase

\section{Acknowledgments}

This work was supported by grants from the Ministry of Science and Technology, Taiwan (MOST 104-2320-B-039-042-MY3) and NSYSU-KMU JOINT RESEARCH PROJECT, (\#NSYSUKMU 106-P006).

\section{Author Contributions}

Y.T.T and C.H.L conceived and designed the experiments; C.Y.K, Y.D.K. and H.C.L. performed the experiments; L.H.W. and C.H.L analyzed the data; C.H.L contributed reagents/materials/analysis tools; C.H.L wrote the paper.

\section{Competing Interests}

The authors have declared that no competing interest exists.

\section{References}

1. Jang JY, Lee JH, Kang BW, Chung KT, Choi YH, Choi BT. Dichloromethane fraction of Cimicifuga heracleifolia decreases the level of melanin synthesis by activating the ERK or AKT signaling pathway in B16F10 cells. Experimental Dermatology 2009; 18: 232-37.

2. Lee HD, Lee WH, Roh E, Seo CS, Son JK, Lee SH, Hwang BY, Jung SH, Han SB, Kim Y. Manassantin A inhibits cAMP-induced melanin production by down-regulating the gene expressions of MITF and tyrosinase in melanocytes. Experimental Dermatology 2011; 20: 761-63.

3. Liu WS, Kuan YD, Chiu KH, Wang WK, Chang FH, Liu CH, Lee CH. The extract of Rhodobacter sphaeroides inhibits melanogenesis through the MEK/ERK signaling pathway. Marine Drugs 2013; 11: 1899-908.

4. Fleischer T, Chang TT, Chiang JH, Sun MF, Yen HR. Improved survival with integration of Chinese herbal medicine therapy in patients with acute myeloid leukemia: a nationwide population-based cohort study. Integrative Cancer Therapies 2016; pii: 1534735416664171.

5. Han R, Tang F, Lu M, Xu C, Hu J, Mei M, Wang H. Protective effects of Astragalus polysaccharides against endothelial dysfunction in hypertrophic rats induced by isoproterenol. International Immunopharmacology 2016; 38: 306-12.

6. Auyeung KK, Han QB, Ko JK. Astragalus membranaceus: A Review of its protection against inflammation and gastrointestinal cancers. The American Journal of Chinese Medicine 2016; 44: 1-22.

7. Chen HW, Lin IH, Chen YJ, Chang KH, Wu MH, Su WH, Huang GC, Lai YL. A novel infusible botanically-derived drug, PG2, for cancer-related fatigue: a phase II double-blind, randomized placebo-controlled study. Clinical \& Investigative Medicine 2012; 35: E1-11.

8. Kuo YL, Chen $\mathrm{CH}$, Chuang $\mathrm{TH}$, et al. Gene expression profiling and pathway network analysis predicts a novel antitumor function for a botanical-derived drug, PG2. Evidence-Based Complementary and Alternative Medicine 2015; 2015: 917345 .

9. Ito S, Nakanishi Y, Valenzuela RK, Brilliant MH, Kolbe L, Wakamatsu K. Usefulness of alkaline hydrogen peroxide oxidation to analyze eumelanin and phenomelanin in various tissue sample: application to chemical analysis of human hair melanins. Pigment Cell \& Melanoma Research 2011; 24: 605-13.

10. Tu, DG, Chang WW, Lin ST, Kuo CY, Tsao YT, Lee CH. Salmonella inhibits tumor angiogenesis by downregulation of vascular endothelial growth factor. Oncotarget 2016; 7: 37513-23.

11. Jang JY, Kim HN, Kim YR, Choi WY, Choi YH, Shin HK, Choi BT, Partially purified components of Nardostachys chinensis suppress melanin synthesis through ERK and Akt signaling pathway with cAMP down-regulation in B16F10 cells. Journal of Ethnopharmacology 2011; 137: 1207-14

12. Ye Y, Wang $\mathrm{H}$, Chu JH, Chou GX, Yu ZL. Activation of p38 MAPK pathway contributes to the melanogenic property of apigenin in B16 cells. Experimental Dermatology 2011; 20: 755-7.

13. Wu LC, Lin $Y Y$, Yang SY, Weng $Y T$, Tsai $Y T$. Antimelanogenic effect of c-phycocyanin through modulation of tyrosinase expression by upregulation of ERK and downregulation of p38 MAPK signal pathways. Journal of Biomedical Science 2011; 18: 74.

14. Choi WJ, Kim M, Park JY, Park TJ, Kang HY. Pleiotrophin inhibits melanogenesis via Erk1/2-MITF signaling in normal human melanocytes. Pigment Cell \& Melanoma Research 2015; 28: 51-60. 